\title{
Ant Colony Optimization for Dynamic Routing in Wireless Computer Networks for Improvement in Quality of Services
}

\author{
Anuj Sharma, PhD. \\ Professor \& Director \\ Institute of Technology Roorkee (ITR, Roorkee)
}

\author{
Mahendra Pratap Panigrahy \\ Associate Professor (ECE) \\ Institute of Technology Roorkee (ITR, Roorkee)
}

\begin{abstract}
Dynamic Routing Assignment (DRA) is a key problem in intelligent optical computer networks. Mathematicians \& Computer Scientists started researching the behavior of ants in the early 1990's to find new routing algorithms. The most efficient \& widest AI techniques like Swarm particle optimization and Ant colony optimization (ACO) are used to find out solutions for dynamic problems. Ant Colony Optimization (ACO) and in the case of well implemented ACO techniques, optimal performance is comparative to existing top-performing routing algorithms. Such a research has yielded ways to minimize the number of nodes that are taken to get to the destination, techniques for quickly resolving an efficient path, and ways to avoid having loops within a routing scheme. Simulation shows that the modified algorithm decreases the blocking probability and increases the resources utilization comparing with the traditional algorithms.
\end{abstract}

\section{Keywords}

Dynamic Routing Assignment, Intelligent wireless computer networks, Ant colony optimization, Blocking probability, Resource Utilization.

\section{INTRODUCTION}

As the Internet and various Internet Protocol based services are changing dramatically, because it highly complex system which comprises and integrates a number of wired and wireless networks covering the needs of different community of users and ranging from small body area networks to global satellite networks, so the traditional computer network is undergoing tremendous changes. Many new interactive and real-time applications have been shown a great challenge for the traditional transport network. There is a British proverb that "all the time- everywhere access" has the meaning of view of pervasive computing that is practice allowing a continual access to data, users, and services. The routing protocols play a very important role in calculating, choosing and selecting the relevant path for transferring the data from the source to the destination efficiently. There are already many accepted routing algorithms to find the shortest path and also to increase the throughput of the network. In this paper, a routing algorithm has been proposed which may be applied in large network (wired and wireless) structure with heavy load. The algorithm proposed here is selecting the path of the data packets guided by the shortest path but with a probabilistic nature. The goal of every network routing algorithm is to direct the traffic from source to the destination maximizing the network performance. The performance measure that is usually taken into account is the throughput (bits delivered per unit time) and number of packets successfully reaching the destination. Here the performance measure is taken as the throughput and also the number of packets successfully reaching the destination, i.e., the reliability of the network. This paper is divided into three major Sections. Section 1 gives the techniques $\&$ problems in routing, in Section 2 there is a brief explanation of the ACO, Section 3 explains how ACO can be applied in the routing problems of the network.

\section{TECHNIQUES FOR INFORMATION TRANSMISSION:}

There are two different techniques currently used by computers to send information across the global internet, Circuit Switching and Packet Switching. Circuit switching is often compared to a telephone call because it follows the same basic principles. The phone is picked up, a call is made to a receiver which is notified, the receiver receives the call and can only talk to one client at a time, and finally when the call is over both the caller and the receiver hang up the phone and end the call. This is the same basic procedure as circuit switching, once a connection has been made between two points that connection is held open for just those points until the call is over. All of the packets of data follow the same path to their destination and they arrive in the same order as they are sent. For the router to be able to set up this kind of connection it must have the knowledge of the entire topology of the network. This requires a lot of information to be stored on the actual router level. The router must work with this data to formulate the best path to send the packets on to the receiver. Packet switching, however, is much less organized. The packets which are sent from one computer to another do not necessarily follow the same path or arrive in the same order. At each different hop that a packet encounters in the network, that router decides which path to send it to next. These two techniques force different requirements to be in place at the router level. Packet switching requires much less state to be stored at the router level than circuit switching, routers only need to know who their neighbors are and how much cost is associated with getting each of them. The packets get routed at each hop and it is not needed for the sending router to know how packets will get to their destination. This scheme does not guarantee that the packets will be received in the same order they were send, and the receiver must be able to handle this occurrence. 


\subsection{Dynamic Routing Techniques for Information transmission:}

The routing scheme in a computer network can be classified into two major categories- Proactive and Reactive. In Proactive the topology of the entire network is maintained and updated on fixed time intervals of a few seconds. All nodes know how to reach each other every instant. But in reactive: the routing path is built every time it is needed and a cache is used for frequently used paths (lazy approach). These algorithms have a characteristic delay every time a packet needs to be sent to a new destination. While a proactive approach allows a fast communication without delays, it requires a constant bandwidth and node's resources overhead on the network to keep the routing table updated. On the other hand it is suitable for those scenarios where all nodes want to communicate between themselves without preferred paths. On the contrary a reactive approach will establish a routing path only when it is needed, limiting the use of resources to the bare minimum. However the cache needs to be very effective to prevent delays caused by new connections or changes in topology. There are so many dynamic algorithms such as AODV (Ad-hoc On-demand Distance Vector), MIT SrcRR, LUNAR - Light Underlay Network Ad-hoc Routing, DSRMonarc, TORA (Temporally Ordered Routing Algorithm), OLSR (Optimized Link State Routing). All the algorithms has some basic advantages and disadvantages. It's essential to decide which algorithms, among the existing ones, were the most suitable for the field of application, a list of criteria has been compiled. This list, also useful to evaluate the maturity of a particular implementation, is available here for reference, compiled without a particular order.

\subsection{Routing Problems in the Computer Networks:}

Ants exhibit the ability to create long bi-directional paths between their homes to their goals in optimal time. These paths may develop obstructions which cannot be crossed and the path may need to be changed at any point in time. By mimicking these abilities with routing algorithms researchers believe they can help the computer network as a whole flow faster. At any given moment there are routers and other routing devices which are taken down for an assortment of reasons. These devices could have been vital nodes for a path given by another router. It is important that the routers which were previous in the path be able to find a new efficient path quickly.

\section{BASIC ACO ALGORITHM:}

The principle of ant colony system algorithm is that a special chemical trail (pheromone) is left on the ground during their trips, which guides the other ants towards the target solution. More pheromone is left when more ants go through the trip, which improved the probability of other's ants choosing this trip. Furthermore, this chemical trail (pheromone) has a decreasing action over time because of evaporation of trail. In addition, the quantity left by ants depends on the number of ants using this trail. Fig. 1 presents a decision-making process of ants choosing their trips. When ants meet at their decisionmaking point $A$, some choose one side and some choose other side randomly. Suppose these ants are crawling at the same speed, those choosing short side arrive at decision-making point $B$ more quickly than those choosing long side. The ants choosing by chance the short side are the first to reach the nest. The short side receives, therefore, pheromone earlier than the long one and this fact increases the probability that further ants select it rather than the long one. As a result, the quantity of pheromone is left with higher speed in short side than long side because more ants choose short side than long side. The number of broken line in Fig. 1 is direct ratio to the number of ant approximately. Artificial ant colony system is made from the principle of ant colony system for solving kinds of optimization problems. Pheromone is the key of the decision-making of ants.

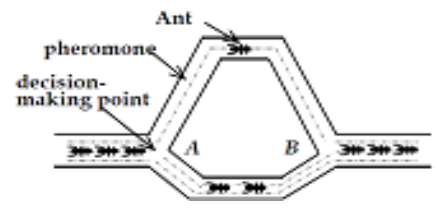

Figure 3.1. A decision making process of ants choosing their trips according to their pheromones

\subsection{Proposed Computational Flow Chart for the ACO Path and Dynamic Routing Environment:}

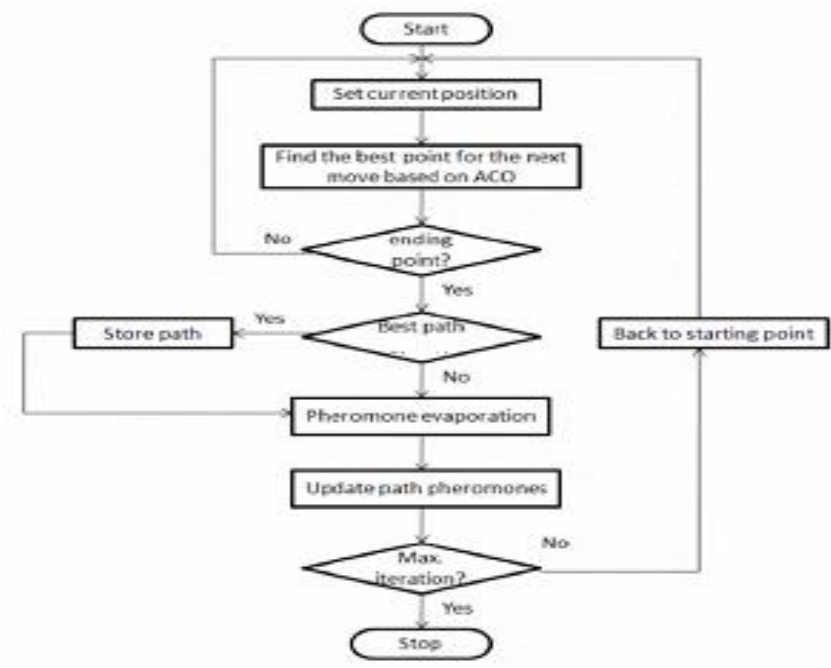

Figure 3.2. Computational flow chart of ACO 


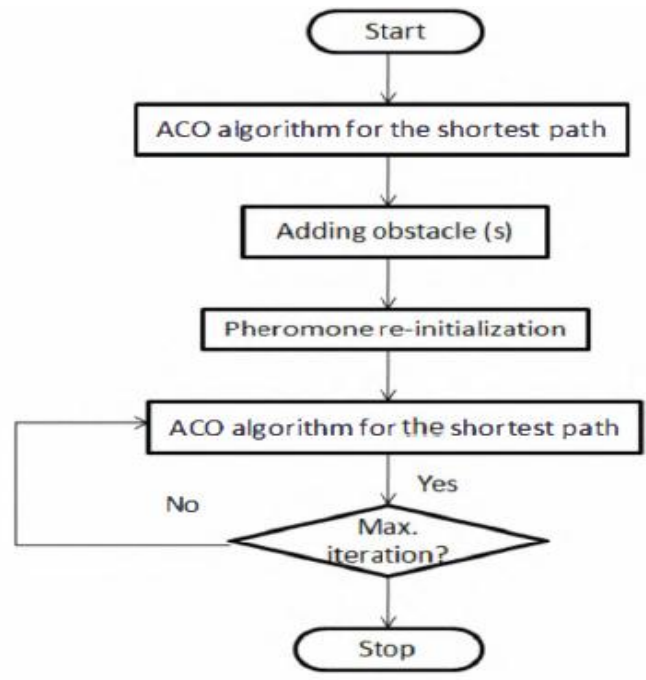

Figure 3.3. The flow chart of ACO in a Dynamic Domain

\subsection{General Ant Colony Pseudo Code:}

if (forward ant)

\{

Get the next node based on the distance

value

if (the link is available and no loop caused) then \{

- Update forward ant with network status

(stack)

- Send forward ant to the next node

else if (no such link exist)

\{

- Create backward ant and load content of forward ant to backward

ant (queue)

- send Backward ant towards source along the same path

as

forward ant

\}

\subsection{Proposed ACO Algorithm and the Mathematical formulations for Shortest Routing:}

The proposed algorithm of ACO has the following concepts:

a. From source nodes to destination nodes the path search ants are called forward ants, they performs the path search function, and establish the reverse pheromone table.

b. As the forward ants reach the destination nodes, from the destination nodes returning to the source nodes, these ants are called backward ants, which performs the pheromone update function, and establish the routing table.

c. In each search process, forward ants reach a certain node to establish the routing table which points to the source nodes and be called reverse pheromone table, which includes the source node, next node, the reverse node pheromone $\tau_{(\mathrm{i}, \mathrm{j})}$.
The proposed steps of the changed ACO is as follows

\section{Step 1: Routing Selection Criteria:}

Suppose each ant represents certain sizes of packets in the link to transfer, and each time the choice of certain path will take up certain size of bandwidth. Ants from source routers set out to the end of router $d$, in each step of the constructed journey, the ant $\mathrm{k}$ chooses rule according to the probability behavior known as a random proportion rule, and to determine the next step will move to which one of router nodes. If the ant $\mathrm{k}$ is currently locating at router $i$, the probability of choosing $j$ as the next visit city is:

$$
p_{i j}^{k}=\frac{\left[\tau_{i j}\right]^{\alpha}\left[\eta_{i j}\right]^{\beta}}{\sum_{i \in N^{k}}\left[\tau_{i l}\right]^{\alpha}\left[\eta_{i l}\right]^{\beta}}, \quad j \in N^{k}
$$

In the expression, $\eta_{\mathrm{ij}}=$ dij- 1 represents an in advance given heuristic information, $\alpha$ and $\beta$ are two parameters, they decides relative influence of pheromone heuristic information, $\mathrm{N}_{\mathrm{ik}}$ represents the set of the adjacent cities that ant k situated in city i can directly arrive, i.e., the collection of all cities that have not been visited by ant $\mathrm{k}$ (the possibility is 0 to choose one from $\mathrm{N}_{\mathrm{ik}}$ cities). Under this probability rule, the probability of choosing some edge $(i, j)$ is decided by the corresponding pheromone $\tau i j$ and heuristic information value $\eta_{\mathrm{ij}}$ of this edge

\section{Step-2: Pheromone Updating Information Criteria:}

When all the ants complete the building of path, that is after reaching the destination router node, the pheromone on each edge will be updated, namely ANT-CYCLE model, and there are also other methods, such as it will update once after the ant passes a node, experiments show that update is better after reaching the destination. First of all, all the pheromone on all edges will reduce the size of a constant factor, and the pheromone will be increased on the edges that ants pass through. The evaporation of pheromone executes according to the following:

$$
\tau_{i j} \leftarrow(1-\rho) \tau_{i j}, \forall(i, j) \in L
$$

Among them $\rho$ is the evaporation rate of pheromone, $0<\delta<1$. The role of $\rho$ parameter is to avoid the pheromone's infinite accumulation, and still can make the algorithm "forget" the selection of poorer path ago, to reduce the occurrence of local optimum. After the evaporation step of pheromone, all the ants release pheromone on the passing edges:

$$
\tau_{i j} \leftarrow \tau_{i j}+\sum_{k=1}^{m} \Delta \tau_{i j}^{k}, \forall(i, j) \in L
$$


Among them, $\Delta \tau^{\mathrm{k}}{ }_{\mathrm{ij}}$ is the pheromone amount that the ant $\mathrm{k}$ releases to the passed edges. $\Delta \tau_{\mathrm{ij}}^{\mathrm{k}}$ is defined as:

$$
\Delta \tau_{i j}^{k}=\left\{\begin{array}{cc}
1 / C^{k}, & \text { if edge }(i, j) \text { is in path } T^{k} \\
0, & \text { otherwise }
\end{array}\right.
$$

Inside the expression, $C^{k}$ the length of path $T^{k}$ that ant $\mathrm{k}$ established, that is the sum of all the length of edges in $T^{k}$. According expression (4), better is the path constructed by ants, more pheromone will be obtained on each edge of the path. Generally speaking, if an edge is selected by more ants, and the total length of the path including this edge is shorter, thus this edge will get more pheromone, and in the later iteration it is more likely chosen by ants.

\section{Step-3: Error Maintenance in Route:}

Route maintenance is accomplished through the use of route error packets and acknowledgments. Route error packets are generated at a node when the data link layer encounters a transmission problem. When a route error packet is received, the hop in error is removed from the node's route cache and all routes containing the hop are truncated at that point. In addition to route error messages, acknowledgments are used to verify the correct operation of the route links. Such acknowledgments include passive acknowledgments, where a mobile is able to hear the next hop forwarding the packet along the route. In Ant Dynamic Source Routing (ADSR) the Forward Ant (FANT) and backward ant (BANT) packets are added in the route request and route reply of DSR respectively as shown in format sequence 1 and 2. FANT and BANT packets are used in this route discovery process.

IP Header $\rightarrow$ DSR Fixed Header $\rightarrow$ Source Address $\rightarrow$ Sequence Number $\rightarrow$ Destination Number $\rightarrow$ Delay Energy Jitter $\rightarrow$ Route Record $\rightarrow$ Hop Count $\rightarrow$ Route Address Addl, Add2...Addn

Format Sequence 1: For the ADSR Route Request Packet

IP Header $\rightarrow$ DSR Fixed Header $\rightarrow$ Source Address $\rightarrow$ Destination Address $\rightarrow$ Delay Energy Jitter $\rightarrow$ Dynamic Route Record $\rightarrow$ Sequence Number $\rightarrow$ Reply Source Address Add1, Add2...Addn

Format Sequence 2: For ADSR Route Reply Packet format

Forward ants are used to explore new paths in the network. Ants measure the current network state for instance by trip times, hop count or Euclidean distance traveled. Backward ants serve the purpose of informing the originating node about the information collected by the forward ant. The ant routing has two types of feedback: positive feedback increases the pheromone levels on routes actively carrying ant packets and negative feedback periodically decreases pheromone values to limit the effects of stale information. Routing decisions tend to support paths with higher pheromone levels and, when allowed to converge, shortest end-to-end paths are empirically observed to be favored. Modified ant mechanism algorithm that uses energy, delay and jitter metrics to perform updates of pheromone levels is proposed. Assuming a control packet containing energy, delay and jitter metrics, a separate pheromone level will be maintained for each metric.

In the algorithm, ant packet headers have fields that:

1. Track the minimum residual energy of the nodes that relay them and

2. Track the cumulative delay and jitter based on backlog information of queued packets destined to the packet' s source.

Thus, energy, delay and jitter pheromone levels will be maintained at each node.

\section{SIMULATION RESULTS, INTERPRETATION \& PERFORMANCE EVALUATION:}

The Figures 4.1 shows the effect of end-to-end average delay for the AODV, OLSR and ACOA. The Figures 4.2 shows the Pause Time for 100, $150 \& 200$ nodes for delivery. In case of AODV \& OLSR there are possibilities that some links might be shared by more than one shortest path. This will increase traffic on selected links which may lead to congestion and hence data packets transmitted through these links may face additional delay and leads to the pause time delay. The packets loss while transmission is also recorded in Figure 4.3. All the three figures give the complete ideas of the imposed algorithm with a improved performance. Performance can further be improved.

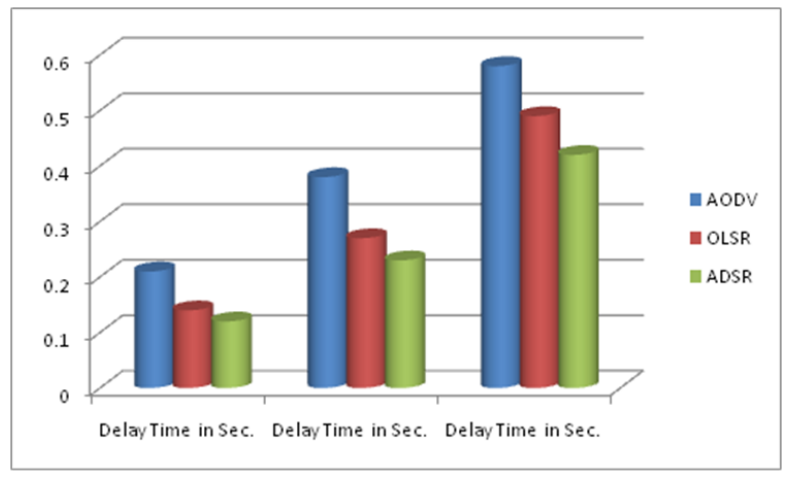

Figure 4.1: Delay Time for $20,40 \& 60$ notes in seconds

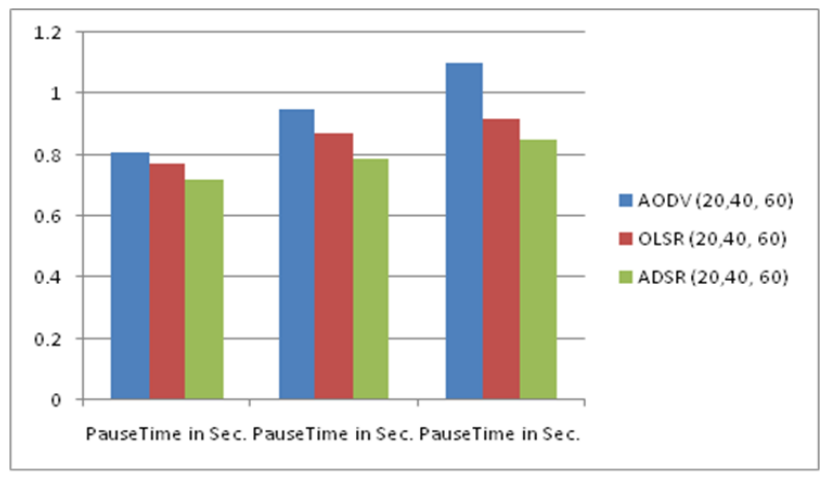

Figure 4.2: Pause Time for 20, $40 \& 60$ nodes in seconds for delivery 


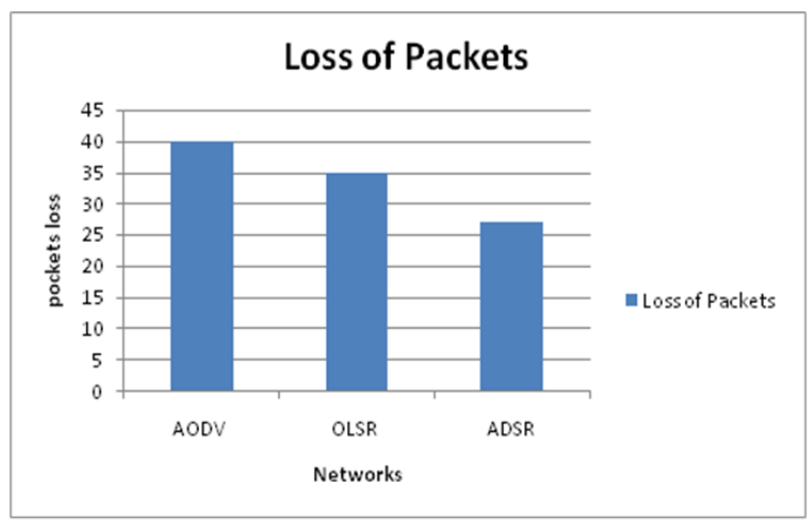

Figure 4.3: Packets Loss while transmission

\section{CONCLUSION:}

This article aiming to the flow distribution problem on the links of network, based on ant colony algorithm adding reverse feedback mechanism, proposes an improved algorithm to solve the network load dynamic balance. Simulation results show that this method can judge the flow on the network links, allocate the flow to reach the load balance and improve the network efficiency. Future work will focus on the research of balance performance of complex network simulation. By refereeing the simulation results, we can see that the new ant colony based algorithm can outperform the comparison schemes and approaches with exhaustive search method. Moreover, this algorithm is efficient in sense that the number of ants used for the forward propagation is always equal to the number of ants used for the backward propagation. So such a network gives the steady performance.

\section{REFERENCE:}

[1] Banik, Bibhash Roy, Biswajit Saha and Nabendu Chaki, "Design of QoS Routing Framework based on OLSR Protocol," ARTCOM 2010, Kochin, Kottyam Kerala, IEEE Explorer, pp-171-73, 2010.

[2] L Chen, WB Heinzelman, A survey of routing protocols that support QoS in mobile ad hoc networks. IEEE Netw 21(6), 30-38 (2007).

[3] L Hanzo II., R Tafazolli, A survey of QoS routing solutions for mobile ad hoc networks. IEEE Common Surv Tutor 9(2), 50-70 (2007).
[4] P Deepalakshmi, S Radhakrishnan, Ant colony based QoS routing algorithm for mobile ad hoc networks. Int J Recent Trends Eng 1(1), 459-462 (2009).

[5] M Dorigo, G Di Caro, The ant colony meta-heuristic. in New Ideas in Optimization, ed. by Corne D, Dorigo M, Golver F (McGraw-Hill, 1999), pp. 11-32.

[6] J. G. Vlachogiannis, N. D. Hatziargyriou, and K. Y. Lee, "Ant Colony System-based Algorithm for Constrained Load Flow Problem," IEEE Trans on Power Systems, pp. 1241-1249, 2005.

[7] Vittorio Maniezzo, Luca Maria Gambarde, Fabio de Luigi. http://www.cs.unibo.it/ bison/ publications / ACO.pdf

[8] "Ant colonies for the traveling salesman problem" http://www.idsia.ch/ luca/acs-bio97.pdf.

[9] International Journal of Computer Application (09758887) No.4, August 2010: "Comparative Analysis Of ACO and Swarm Optimization Techniques", V.Selvi lec, dept. Of CSE, Nehru Memorial College, Trichy and Dr.R.Umarani Associative Professor, dept of CSE, saratha college of women, salem.

[10] KASSABALIDIS I, El-SHARKAWI M A, MARKS R J. Swarm Intelligence for routing in communication networks. Global Telecommunications, 2001,6(6):36133617.

[11] Brian Hill, "The Complete Reference, CISCO”, TATA MCGRAW-Hill publishing Ltd., N.Delhi.

[12] Ant algorithms for discrete optimization. Source, Artificial Life archive. Volume 5 , Issue 2 (April 1999).

[13] O. Cordon, F. Herrera, and T. Stutzle, Special Issue on Ant Colony Optimization: Models and Applications, Mathware and Soft Computing, vol. 9, Dec. 2002.

[14] Ant Colonies for Adaptive Routing in Packet-Switched Communications Networks. Source, Lecture Notes In Computer Science.

[15] AntNet:ACO routing algorithm in practice: Vincent Verstraete, Matthias Strobbe, Erik Van. 